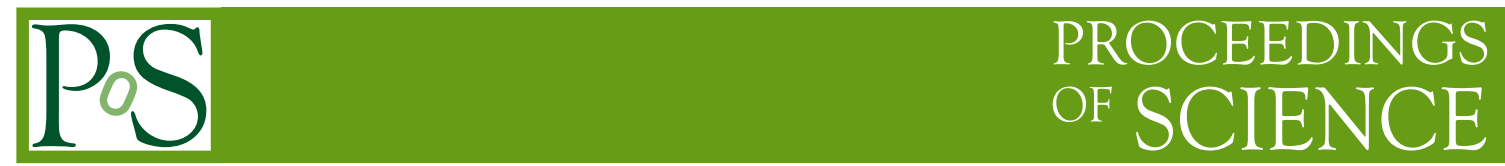

\title{
Search for Muon to Electron Conversion at J-PARC
}

\section{Satoshi Mihara*t}

KEK-IPNS/J-PARC Center/Sokendai

E-mail: satoshi.mihara@kek.jp

The COMET experiment aims to search for the the lepton-flavor violating muon reaction, mu-e conversion, with sensitivity better than $10^{-14}$ in Phase I and $10^{-16}$ in Phase II. We present the status and prospect of the experiment.

XIV International Conference on Heavy Quarks and Leptons (HQL2018)

May 27- June 1, 2018

Yamagata Terrsa, Yamagata,Japan

* Speaker.

${ }^{\dagger}$ on behalf of the COMET collaboration. 


\section{Introduction}

Muon has been playing an important role in early stage of establishing the Standard Model (SM) of the elementary particle physics. Because the muon decay to electron(s) without any neutrino in the final state had not been observed in any experiment[1] and the muon neutrino does not produce an electron in its interaction, i.e. the muon neutrino is different from the electron neutrino[2], the concept of the lepton flavor is derived and the lepton flavor number is found to be naturally conserved in the framework of the SM. Quest for the muon lepton flavor violation has been conducted along with establishing the SM; intensive studies were carried out especially after three Meson Factories were constructed at TRIUMF in Canada, LANL in the US, and SIN in Switzerland (currently named as PSI) in 1970s. All experiments conducted in these facilities were motivated by the possible existence of new physics beyond the SM, aiming at detecting a hint of it. Despite continuous efforts to find a signal of lepton flavor number violation, no evidence nor indication has been found yet. Nevertheless motivation to search for the lepton flavor number violation is stronger than ever as the search is thought to be complementary to the high-energy frontier experiments at the Large Hadron Collider (LHC) where direct searches of new physics signals are intensively conducted.

Many theoretical models beyond the Standard Model have predicted existence of three muon reactions violating the lepton flavor number conservation; $\mu \rightarrow e \gamma, \mu \rightarrow e e e$, and $\mu-e$ conversion [3]. These reactions have different sensitivities to different physics cases depending on what kind of new particle contributes in the reaction. Experimental discovery of any reaction would clearly indicate the existence of new physics as the lepton flavor violating process is strictly forbidden in the SM. However it is not straightforward to extract information of new physics only from a single reaction process even if the clear signal is observed. This is because the process is caused by new particle(s) and interaction(s) contributing only in intermediate state of the reaction. Thus it is quite important to carry out three searches with similar physics sensitivities and compare the results to extract detailed information of new physics behind them.

Among these three muon processes, $\mu \rightarrow e \gamma$ and $\mu \rightarrow e e e$ searches should be carried out using continuous muon beam as there are more than one particles in the final states and the experiment sensitivity could be potentially limited by accidental overlaps of particles from different reactions. On the other hand $\mu-e$ conversion search should be carried out using pulsed muon beam with a suitable pulse timing structure to remove the prompt background. It is also important to handle environmental background such as cosmic rays in the $\mu-e$ conversion search.

The $\mu-e$ conversion is expected to occur by coherent muon interaction with quarks contained in the nucleus after a muon is trapped in a nucleus ( muonic atom formation ) and transits to the $1 \mathrm{~S}$ state. The muon life time in a muon atom is shorter than that in the free space because muon capture process $\left(\mu^{-}+(A, Z) \rightarrow v_{\mu}+(A, Z-1)\right.$ where $A$ and $Z$ show mass number and atomic numbers of the target nucleus) contributes. For the case of aluminium is used as a muon stopping target to form muonic atoms, the life time of muons $\left(\tau_{\mu}^{N}\right)$ is 860 nanoseconds. The pulse timing structure of the muon beam for the $\mu-e$ conversion search should be in the similar timing scale to $\tau_{\mu}^{N}$. This indicates that the proton beam time structure producing the muon beam should also be in the similar timing scale as muons are produced as decay products of promptly produced pions in proton-nucleus interactions. It is also quite important that the proton beam pulse should 
be precise, namely the leakage of protons from a pulse to in-between pulses (the ratio is called 'beam extinction factor') should be minimized below the level required by the experiment. This is because such protons can produce pions in a delayed timing and such pions can produce energetic electrons that can be misidentified as the $\mu-e$ conversion signal electron. An example of pulsed proton beam time structure for a $\mu-e$ conversion search experiment is illustrated in Figure 1. The data acquisition system is activated a few hundreds nanoseconds after the proton beam hits the pion production target in order to sufficiently suppress the background caused by prompt pions.

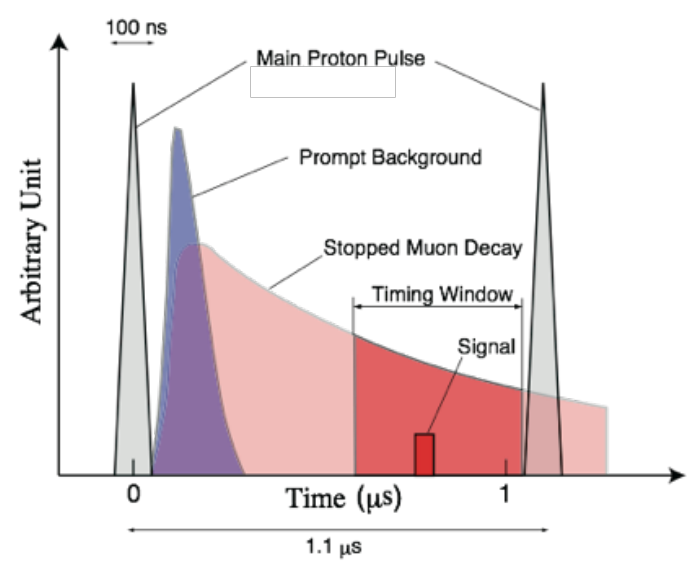

Figure 1: An example of pulsed proton beam time structure for an $\mu-e$ conversion search experiment. The COMET experiment at J-PARC employs a proton beam structure of 1.1 microseconds pulse repetition rate to produce the pulsed muon beam.

In the $\mu-e$ conversion process only single electron is emitted in the final state without any neutrino emission, providing electron energy of $m_{\mu}-B_{\mu}-E_{\text {recoil }}$ where $m_{\mu}, B_{\mu}$, and $E_{\text {recoil }}$ are muon mass, muon binding energy in the muonic atom, and recoil energy, respectively. In case aluminium is used to form muonic atoms the signal electron energy will be $104.97 \mathrm{MeV}$, which is above the tail of decay-in-orbit (DIO) electron energy spectrum. Thus the signal of the $\mu-e$ conversion can be identified with this characteristic energy and delayed timing.

The current best sensitivity to search for the $\mu-e$ conversion was achieved by the SINDRUM II experiment conducted at PSI [4]. They set the upper limit of $7 \times 10^{-13}$ at the $90 \%$ confidence level.

\section{COMET experiment at J-PARC}

The COMET experiment at J-PARC aims to search for the $\mu-e$ conversion with 10,000 times better sensitivity than the current limit achieved by the SINDRUM II experiment in two phases; Phase I and Phase II. In COMET Phase I the single event sensitivity better than $10^{-14}$ is anticipated by using a pulsed proton beam of $3.2 \mathrm{~kW}$ provided by the J-PARC accelerator [5]. A schematic view of the experimental setup is presented in Figure 2 (left). The COMET phase II will follow Phase I with 18 times larger beam power of $56 \mathrm{~kW}$ and with extending the chain of solenoid magnet as shown in Figure 2 (right) for better beam background suppression and higher reduction rate of background electrons entering the detector system. The Phase II plans to achieve the single event 
sensitivity better than $10^{-16}$ with this setup. The major background source would be the cosmic-ray as long as the beam extinction factor can be suppressed below $10^{-11}$.
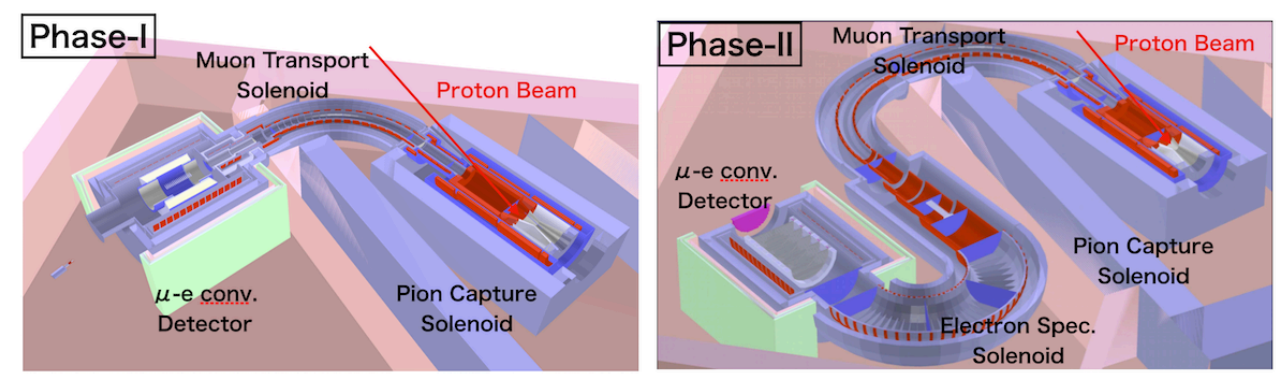

Figure 2: Layout of the COMET experiment Phase I (left) and Phase II (right).

The proton beam for the COMET experiment is provided by the J-PARC accelerator composed of $400 \mathrm{MeV}$ linear accelerator (LINAC), $3 \mathrm{GeV}$ Rapid Cycle Synchrotron (RCS), and the Main Ring (MR). The MR is usually operated at $30 \mathrm{GeV}$ to provide proton beam to the neutrino experiment (T2K) and nuclear and particle physics experiments in the Hadron Hall. Instead COMET utilizes the proton beam of $8 \mathrm{GeV}$ to suppress anti-proton production in the proton-nucleus interaction. The proton beam extinction factor should be better than $10^{-11}$ so that COMET can achieve the target sensitivity better than $10^{-16}$ in Phase II without being suffered from beam related background.

The required time structure of the proton beam is realized by filling protons in every two acceleration buckets of the MR and extracting them with keeping the bunch structure. The extracted proton beam is transported to the experiment area using conventional beam transport elements. The proton beam is then injected to a pion production target located at the center of a capture solenoid of gradient magnetic field. COMET utilizes pions and muons produced backward by transporting them to the experiment area using a curved solenoid. A layout of proton beam transport elements is shown in Figure 3.

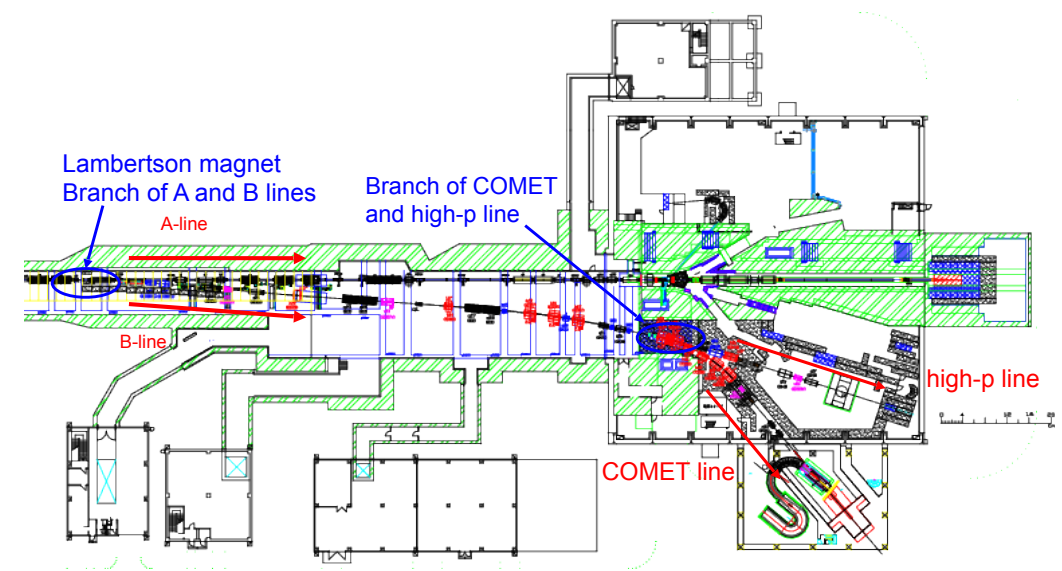

Figure 3: Layout of proton beam transport system for the COMET experiment 
COMET Phase I intends to achieve the $\mu-e$ conversion search sensitivity better than $10^{-14}$ using $3.2 \mathrm{~kW}$ proton beam injected to a graphite target. The muon stopping target composed of 100 micrometer thick aluminium disks is located after the first 90 degree curved solenoid as shown in Figure 2 (left). The collimator system will be equipped in another short solenoid magnet, bridge solenoid, connecting the magnetic field of the curved solenoid and detector solenoid smoothly.

Construction of the solenoid magnet system including the cryogenics is ongoing; the curved solenoid is already installed in the experiment area with a sufficient alignment precision and being tested, individual coil winding composing the capture solenoid is in progress to be assembled before installing into a cryostat, and fabrication of the detector and bridge solenoid magnets is in progress on schedule.

Two detector systems are prepared for COMET Phase I. One is called 'CyDet detector' (Figure 4 (left)) and is used to conduct physics data acquisition, composed of the Cylindrical Drift Chamber (CDC) and trigger hodoscopes installed in the detector solenoid. The muon stopping aluminum disks are located at the center of the CDC. Electron momentum from the target is precisely measured with the CDC with a momentum resolution better than $0.2 \%$ in sigma to suppress the DIO background below $10^{-16}$. The other system is used to measure and understand the newly fabricated muon beam, called 'StrEcal detector' (Figure 4 (right)). StrEcal detector is composed of five straw-tube tracker modules to measure the beam particle momenta, and LYSO crystal calorimeters to measure the energy. R\&D of detector components are almost completed and construction is in progress by the collaboration.
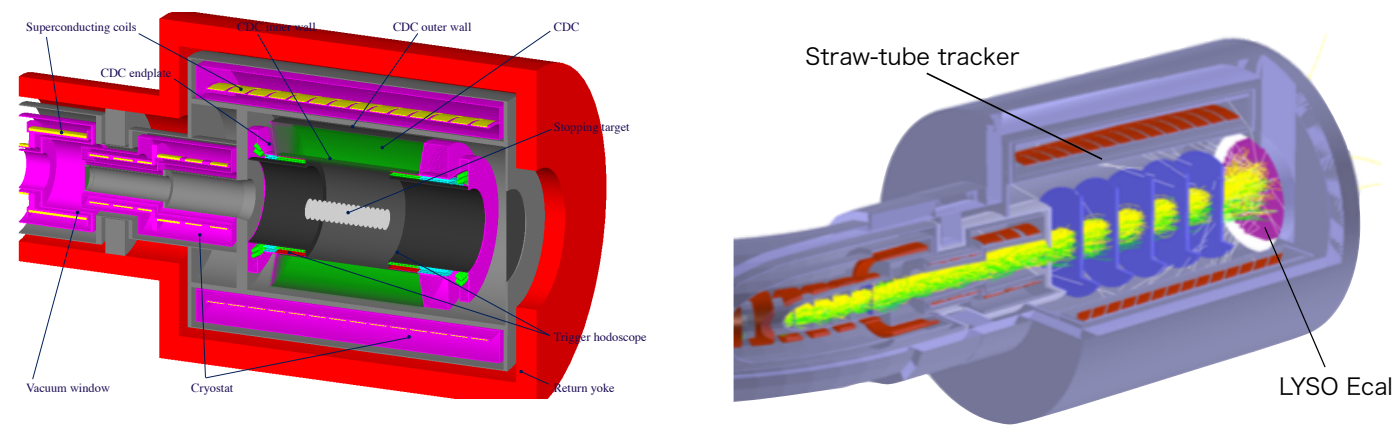

Figure 4: COMET Phase-I detector systems. CyDet detector (left) and StrEcal detector (right).

As already mentioned above, COMET uses $8 \mathrm{GeV}$ proton beam extracted from the MR with a dedicated extraction method. As this operation mode had never been tested at J-PARC, acceleration and extraction tests were conducted in January and February 2018 for the first time. The beam extinction factor measurement was also carried out using the secondary beam produced by the extracted protons. In these tests it has been found that $3.2 \mathrm{~kW} 8 \mathrm{GeV}$ proton acceleration and extraction can be done without any significant beam loss and the beam extinction factor is good enough except beam particle leakage from one of the acceleration bucket to in-between pulses only in first 100 milliseconds after the start of beam extraction. 


\section{Summary}

The COMET experiment at J-PARC aims to search for the lepton-flavor violating muon reaction $\mu-e$ conversion with a sensitivity better than $10^{-16}$. COMET takes a staged approach to realize this sensitivity, Phase I \& II. The solenoid magnet system is constructed down to the end of the first 90 degree bend of the transport solenoid in Phase I. The Phase-I detector system is attached to the end of the transport solenoid. The system is extended in Phase II to receive about 18 times larger proton beam power and to realize better background suppression from the muon stopping target. Preparation of COMET Phase-I is in progress smoothly in all aspects; construction of new proton transport line, solenoid magnet system including the cryogenics, and detector systems to carry out physics and beam measurements.

\section{References}

[1] S. Parker, H. L. Anderson and C. Rey, Search for the decay $\mu^{+} \rightarrow e^{+}+\gamma$, Phys. Rev. 133 (1964) B768.

[2] G. Danby, J.-M. Gaillard, K. Goulianos, L. M. Lederman, N. Mistry, M. Schwartz et al., Observation of high-energy neutrino reactions and the existence of two kinds of neutrinos, Phys. Rev. Lett. 9 (1962) 36.

[3] S. Mihara, J. Miller, P. Paradisi and G. Piredda, Charged lepton flavor-violation experiments, Annual Review of Nuclear and Particle Science 63 (2013) 531

[https://doi.org/10.1146/annurev-nucl-102912-144530].

[4] SINDRUM II collaboration, W. H. Bertl et al., A Search for muon to electron conversion in muonic gold, Eur. Phys. J. C47 (2006) 337.

[5] COMET collaboration, Technical Design Report for COMET Phase I, . 